\title{
Using CAD to Shape Experiments in Molecular QCA
}

\author{
Michael Niemier \\ Georgia Inst. of Tech. \\ 801 Atlantic Drive \\ Atlanta, GA 30332 \\ mniemier@cc.gatech.edu
}

\author{
Michael Crocker, X. \\ Sharon $\mathrm{Hu}$ \\ U. of Notre Dame \\ 384 Fitzpatrick Hall \\ Notre Dame, IN 46556 \\ mcrocker,shu@cse.nd.edu
}

\author{
Marya Lieberman \\ U. of Notre Dame \\ 271 Stepan Chemistry \\ Notre Dame, IN 46556 \\ mlieberm@nd.edu
}

\begin{abstract}
This paper examines how circuits and systems made from molecular QCA devices might function. Our design constraints are "chemically reasonable" in that we consider the characteristics and dimensions of devices and scaffoldings (circuit boards to attach devices to) that have actually been fabricated (currently in isolation). We will show that not only is the work presented here a necessary first step for any work in QCA CAD, but also that by considering issues related to design can actually help shape experiments in the physical sciences for emerging, nano-scale devices. Our work shows that circuits, scaffoldings, substrates, and devices must all be considered simultaneously. Otherwise, there is a very real possibility that the devices and scaffoldings that are eventually manufactured will result in devices that only work in isolation. This work is especially timely as experimentalists are currently working to merge the different experimental tracks - i.e. to selectively place a QCA device.
\end{abstract}

\section{INTRODUCTION}

It is hardly necessary to discuss why it is important to look at computational systems that are not based exclusively on CMOS transistors. Emerging technologies have the potential to offer their own unique "wins". Quantum computation (not the same as QCA) can potentially factor very large numbers efficiently, arrays of nanowires might offer very dense memory, and spin-based systems could provide nonvolatile storage and low power operation.

At least at the device-level, molecular Quantum-dot Cellular Automata (QCA) cells could overcome the obstacles of interconnect and power dissipation that molecular transistors cannot. QCA [20] accomplishes logical operations and data movement via Coulombic interaction rather than electric current flow. A theoretical analysis of a molecular implementation has shown that QCA-based circuits could be clocked at an extremely high frequency (adiabatically at $1 \mathrm{THz}$ [18, 2, 13]), potentially lead to circuits with den-

Permission to make digital or hard copies of all or part of this work for personal or classroom use is granted without fee provided that copies are not made or distributed for profit or commercial advantage and that copies bear this notice and the full citation on the first page. To copy otherwise, to republish, to post on servers or to redistribute to lists, requires prior specific permission and/or a fee.

ICCAD'06 November 5-9, 2006, San Jose, CA

Copyright 2006 ACM 1-59593-389-1/06/0011 ...\$5.00. sities that are 3-4 orders of magnitude beyond what endof-the-curve CMOS can provide [4], and should dissipate very little power $\left(100 \mathrm{~W}\right.$ if $10^{12}$ devices in $1 \mathrm{~cm}^{2}$ switch simultaneously) [18]. Experiments have shown a molecule is capable of switching between configurations that represent binary 0 and 1 states. This work looks at systems of molecular QCA devices - but within the context of chemically reasonable constraints, associated with fabricated candidate devices and scaffoldings.

QCA cells interact as the charge configuration of one cell alters the charge configuration of the next cell. As electrostatic interactions are strongly distance-dependent, the smaller the QCA devices can be made, the better they work. Also, information transmission and processing is carried out by the same entities - QCA cells - rather than by separate devices and wires. In CMOS, the preponderance of real estate on chip is taken up by wires, so shrinking CMOS devices does not necessarily produce corresponding gains in device density. When smaller QCA cells are made, QCA interconnect shrinks too.

Previous work presented at ICCAD has addressed implementable interconnect for molecular QCA. The authors of [4] looked at self-assembling systems based on the DNA tiles proposed by Seeman and Winfree. According to [4], one consequence of this fabrication mechanism was that a co-planar wire crossings would be difficult to fabricate. The work of [4] was important as it showed that an alternative interconnection mechanism did not adversely affect area. As a result, the milestone of fabricating a co-planar wire crossing could be removed from the physical science critical path.

The work presented here is related to the solution discussed in [4], but takes an important step back. In this paper we consider via a statistical mechanical analysis not just whether or not the structures required for the methods proposed in [4] will function properly, but whether or not a functionally complete logic set will function properly if existing devices and potential scaffoldings are used to build it. It will also lay the foundation for any other future work in QCA CAD - helping to quantify important metrics such as wire pitch, the number of devices that can be active simultaneously, the types of defect tolerance required, etc.

Finally, the results of our work will also show that a comment that is sometimes directed at design work relating to any emergent nano-scale device - that "it is premature to consider any design-related work until devices and substrates have been experimentally realized" - is itself misguided. Devices and scaffoldings are not the only milestones on the critical path to a realized, nano-scale system (the de- 
sired end result). If architectures, viable interconnect, defect tolerance, etc. cannot be addressed, the end goal of a system is still not met.

In this paper, we will show that if molecular QCA devices and scaffoldings are in fact considered independently of circuits and systems, we could end up with a combination of devices and scaffoldings that look like circuits, but do not function like circuits. Without a close coupling between all 3 threads (circuits, scaffoldings, and devices), work in any individual thread will be fundamentally incomplete.

By considering how devices and scaffoldings will join to form logic, the design community has the opportunity to push back on physical science - quite possibly having a direct effect on actual experiments. CAD can guide experimentalists with regard to how devices should be made, what their dimensions ideally should be to facilitate logic, circuits, and functional units, what scaffolding capabilities should be targeted, and what device-level redundancy is required.

We will begin in Section 2, by discussing the QCA concept, a molecular implementation of it, and a possible way to pattern individual devices. In Section 3, we will discuss our statistical mechanics-based simulation engine. In Section 4, we present simulation results that look at fundamental logic structures needed for a functionally complete logic set. In Section 5 we consider logic in the context of the device of $[9,19]$ and the DNA-based scaffolding discussed in [6]. We conclude in Section 6.

\section{OVERVIEW OF MOLECULAR QCA}

\subsection{QCA Fundamentals}

QCA represents information by encoding binary numbers in cells that have a bi-stable charge configuration. A QCA cell can consist of 2 or 4 "charge containers" (i.e. quantum dots) and 1 or 2 excess charges, respectively. One configuration of charge represents a binary ' 1 ', the other a binary '0' (Fig. 1a) [20, 11]. Fig. 1b-d illustrates a fundamental set of QCA circuit elements [20]. The majority gate (Fig. 1b) implements the logic equation $A B+B C+A C$. The output cell assumes the polarization of the majority of the 3 input cells [11]. Note that permanently setting 1 input of a majority gate to a 0 or a 1 yields an AND or OR gate respectively; signal inversion is also possible [20] (see Fig. 1e) - hence we can form a functionally complete logic set. A QCA wire (Fig. 1c) is just a line of QCA cells; at the input end, one cell must be polarized to act as the driver for the wire. QCA wires with different orientations (Fig. 1d) can theoretically cross in the plane without the destruction of the binary value on either wire. A signal on a 45-degree wire will alternate between a 1 and 0 ; values can be transferred between wire types ${ }^{1}$.

A "clock" structure turns devices on/off and provides gain. The clock causes groups of QCA cells to transition from a null state (off) to a bistable, active state (1 or 0 ), and then back to a null state. For systems of molecular QCA cells, the clock would essentially be a continuously propagating electric field that would move a computation across a layer of QCA devices. Computation would occur at the leading

\footnotetext{
${ }^{1}[3]$ and our simulations show that by adding redundancy at the device level, the constructs in Fig. 1 should be more resilient to errors/defects (i.e. using a wire that is 2 cells thick instead of 1 cell thick).
}

edge of a wave front as a cell is turned on. The electric field pushes charge up to the active state (providing gain) while a driver - a cell that is already on and polarized - determines if the cell just turned on will be a 1 or 0 . The phases of a clock signal could take the form of time-varying, repetitious voltages applied to CMOS wires embedded underneath a QCA circuit. Four wires - associated with four clock phases - are required in order to generate smooth and continuous field propagation [5]. A different bit of data could be associated with every four wire group. Cells are not clocked individually. From an architectural perspective, this results in a certain amount of inherent pipelining determined by the granularity of the clock structure $[11,12]$.

\subsection{Molecular QCA}

\subsubsection{Devices}

QCA devices (and circuits) have actually been made from metal $[1,17]$ and from magnets [8], but can also be made from a single chemical molecule. Molecular QCA cells correspond to a class of compounds called mixed-valence compounds, which contain multiple redox centers in different oxidation states. For a molecular QCA device, each "quantum dot" or "charge container" would be a single redox center, and the redox centers would be rigidly held together by covalent bonds [14]. A recent experiment demonstrates that applying reasonable electric fields (like from a clock structure) can move a charge between two sites of a molecule engineered to function as a two-dot QCA cell [14]. Thus, experimental results show molecules that act as QCA devices, switching between a chemical representation of a binary 0 and a binary 1 . This is important both as a basic demonstration that molecules can be used as containers for mobile charges, and as a prototype for the clocking of QCA molecules by pulling charges down to a null position from an active QCA layer. While a four-dot cell can essentially be "made" from two-dot cells, four-dot QCA molecules have also been made [9]. Our work will look at logic in the context of 4-dot QCA molecules.

\subsubsection{Making Circuits}

The requirements for positioning molecular QCA cells are stringent. Individual molecules must be placed in specific locations with nanometer precision. This work will begin to explain exactly what precision is necessary to make QCA circuits. Recent results in DNA tiling suggest that DNA could be used as a "circuitboard" to position QCA cells. One viable target assumes DNA tiles fabricated by Seeman and Winfree [23] as the scaffolding. QCA molecules would attach covalently to modified DNA nucleotides. Assuming the tiles retain the B-DNA duplex, sites in the major groove could serve as attachment points with a periodicity of 3.6 Angstroms along the helix axis, and 2 or 4 Angstroms perpendicular to the helix axis (resulting in device densities of $10^{13} / \mathrm{cm}^{2}$.) This work has significant critical mass as many research groups are currently looking at creating and attaching nano-particles to DNA-based scaffoldings (i.e. [10, 16, $15])$.

Thus, DNA has the potential to (1) act as a circuitboard, (2) be made large enough to hold computationally interesting circuit components, and (3) be built with reasonable yields. It should also be possible to enforce extremely rigid cell orientation on the DNA tile - which is good for a 4-dot 


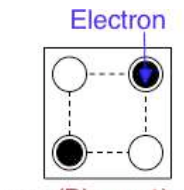

(a.) (Binary 1)

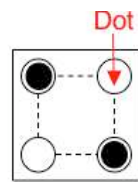

(Binary 0)

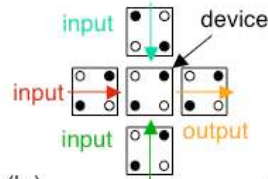

(b.)

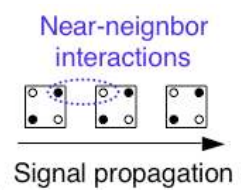

(c.)
Signal propagation

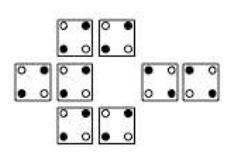

(d.)

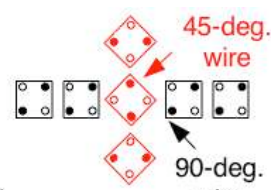

(e.)

wire

Figure 1: "Cartoon" representations of (a) a 4-dot QCA cell, (b) a majority gate, (c) a wire, (d) a QCA physical wire crossing.

molecular QCA cell. There are good precedents for rigid placements of metal complexes on duplex DNA [7].

\subsubsection{Guided Self-Assembly for Systems}

Large DNA structures with molecules attached to them could be "guided" to certain places to form more complex circuits. Directed assembly envisions using 10-100 nm, topdown lithography to guide the attachment of circuitboards to silicon substrates. Interactions between the circuitboards and ordered pads with matching shapes will allow them to dock at certain locations on a surface, with specific partners. DNA hybridization between circuitboards will maintain sub$\mathrm{nm}$ registry from one circuitboard to the next. [6] shows that silicon is a viable substrate for guided DNA tile binding.

\subsection{Fabrication Challenges}

We do note that fabricatable interconnect presents nearto-midterm challenges to system designers. While enforcing one cell orientation appears possible [7], creating a circuit part that has cells with two different rotations (i.e. on the same DNA tile), is a much more difficult task to experimentally solve. While not impossible, a first implementation target will probably be a system that has cells with just one orientation. Thus, the coplanar wire crossing shown in Fig. $1 \mathrm{~d}$ would not be available for any design. Still, in the worst case, the selective duplication and logical crossings proposed in [4] to address interconnect should be satisfactory. Here, we consider the parts needed not just for interconnect, but also for a functionally complete logic set - using the chemically reasonably constraints just discussed.

\section{STATISTICAL MECHANICS}

\subsection{Existing Simulators}

Two tools that simulate systems of QCA devices are QCA Designer [21] and M-AQUINAS [3]. Both are physics-based and assume the presence of a clock field. QCA Designer calculates the ground state of a cell by using a two state Hamiltonian. It numerically solves the Schrodinger equation, which returns an eigenenergy for each of two states. The systems evolves toward the ground state (where cells have polarizations such that the energy of the system is minimized) and the lowest eigenenergy is chosen [21]. It assumes a clock with 4 distinct stages [11]. Groups of cells are turned on, switch, and then drive other groups of cells.

The clocking mechanism envisioned for systems of molecular QCA devices is the continuous clocking scheme proposed by Hennessey and Lent. The advantage of this clocking mechanism, (discussed in Section 2.1), is that a cell is turned on and immediately assumes the polarization of its driver - reducing the risk of the a metastable state. MAQUIANS assumes this clocking mechanism. QCA cells are

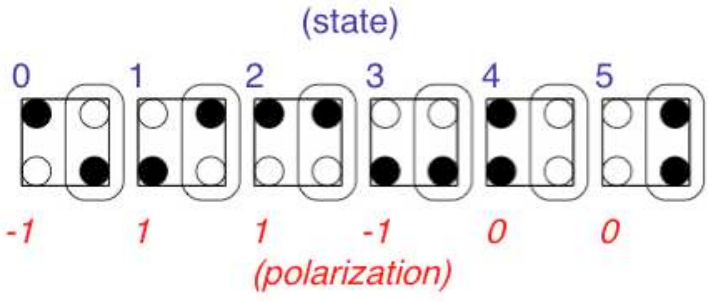

Figure 2: Possible states of a QCA molecule and "seen" polarizations.

simulated in time under the assumption that cell behavior is adiabatic so that the state of the array at time $t$ is very close to the ground state of the array under the influence of the clock frozen at time $t$. At each time of interest, the time independent Schrodinger equation (TISE) is solved for the ground state of each cell in the array. This continues until a self-consistent solution is found for the entire array [3]. The TISE takes into account the electrostatic potential due to both the clock and inter-cellular interactions.

Both simulators allow a user to specify attributes such as cell size, cell spacing, etc.. However, they also only allow a device to be in 3 possible states - a 1 or 0 when the cell is active, or a null state when the device is clocked off. With a molecular device - a.k.a. a 4-dot QCA molecule - there are really 6 possible polarizations (or states) that a device might be in when active (see Fig. 2). Although states 2-5 are higher in energy than states 0 and 1 , they still need to be included for completeness.

It is unlikely that a single, isolated QCA cell would settle into any of the four degenerate cases (2-5 in Fig. 2). With a square molecule, simple trigonometry tells us that the electrons would be farthest from each other when in opposite corners, thus minimizing the total energy of the "system". However, when many QCA cells are placed in close proximity to one another (i.e. to form a circuit), it is possible that a locally higher energy state might be favored because it reduces the energy of the entire QCA circuit - and one of the four degenerate cases might then occur. If this happens in the cell that serves as the output of the circuit (or if a mistake state propagates), it will be impossible to tell whether or not the output is a 1 or a 0 .

\subsection{Statistical Mechanical Analysis}

Our goal is to investigate how well the components needed for a functionally complete logic set and implementable interconnect will function assuming a 4-dot QCA molecule with nanometer dimensions and the scaffolding discussed in Sec. 2. While we will leverage M-AQUINAS (as it supports continuous clocking), our results and analysis are primarily based on statistical mechanical simulations. We have 
modified the work of [22] to consider 4-dot QCA molecules and the 6 possible active states that would be associated with the 4-dot QCA molecule. Our analysis considers electrostatic forces and potentials between all point charges in a circuit. Such calculations are performed for all possible combinations of cell states. The end goal is to use the energy potentials to determine with what probability a circuit will behave correctly, and how "stable" it is.

It is important to note that our simulator does not assume a clock. In a sense, this is the most "pessimistic" simulation possible. In addition to providing gain and preventing metastable states, a continuously propagating clock also prevents some potential metastable states from occurring at all. Certain configurations of cells that could settle into a metastable state will never have the opportunity to interact - as they will never be active simultaneously. However, at the same time, a clock wave is relatively large when compared to cell size. Thus, a column of active cells will be more than one cell wide, and a statistical mechanical analysis is a good way to model this situation. If an unclocked statistical mechanical analysis of a circuit element shows a high probability of the desired logical output, it should certainly function properly in conjunction with a clock.

We cycle through all possible combinations of QCA cell states and sums up the total energy associated with each state. An n-body energy calculation is made between all electrons for each combination. The energy potential between any two electrons $m$ and $n$ is found with Eq. 1.

$$
E_{m, n}=\frac{e^{2}}{4 \pi \epsilon r_{m, n}}
$$

All $6^{N}$ combinations of cell states are considered, leading to a sum of energy potentials for each combination. The sum for each state combination, $i$, is calculated with Eq. 2.

$$
E(i)=\sum_{m=1}^{N-1} \sum_{n=m+1}^{N} E_{m, n},
$$

The combination, $i$, with the lowest energy sum is denoted by $E$ (ground). This ground state combination is used to find the energy difference for each combination. (Each combination, $i$, will be paired with the difference of its total energy, and the total energy of the ground state as shown in Eq. 3. Also note that the $\Delta E$ of the ground state will be 0 .)

$$
\Delta E(i)=E(i)-E(\text { ground }),
$$

Still, it is insufficient to simply determine the ground state. In order to ensure that the behavior demonstrated in the ground state is valid, a stability test must be completed. The result of this stability test is a probability ratio that indicates how likely it is that the ground state behavior will match the overall circuit behavior. The Boltzman equation provides a useful stability measure. For each of the $6^{N}$ combinations there is an energy difference $\Delta E(i)$, which is necessary for the Boltzman energy calculation. (As excited states can be thermally populated we incorporate temperature into our statistical mechanical analysis). By summing the Boltzman energies for all $6^{N}$ combinations, the partition sum $F_{\text {total }}$ can be found. This partition sum is the first part of the probability ratio.

$$
F_{\text {total }}=\sum_{i=1}^{6^{N}} e^{\frac{-\Delta E(i)}{k T}},
$$

A similar sum is calculated for each of the 6 possible outputs (i.e. the states in Fig. 2). The output cell of each combination has a specific state, $j$, between 0 and 5 . All combinations with the same output cell state are grouped together. Next, the same Boltzman calculation is performed for all combinations in the same group, and the sum is kept as $F_{j}$. This is done for all output states, $j=0-5$.

$$
F_{j}=\sum_{i=1}^{6^{N}} e^{\frac{-\Delta E_{j}(i)}{k T}},
$$

Now that we have the Boltzman sum for all combinations, $F_{\text {total }}$, and the Boltzman sums for each possible output cell state, $F_{j}$, the stability ratio can be computed for the ground state. Using the output cell of the ground state, the probability is calculated. If the output cell state is $k$, then the probability that the ground state's behavior will match the overall circuit behavior is found by the ratio of $F_{k}$ to $F_{\text {total }}$. The ratio is calculated for all $k$.

It is also useful to know the output cell's expected value. We can calculate this by using the Boltzman partition sums and the output polarizations of a given cell state (see Fig. 2 ). Note that the polarization value is defined as the difference between the rightmost occupancy of the top and bottom electron wells of the cell. The sums $F_{j}$ are weighted with the corresponding polarization value, summed, and divided by the total partition sum, $F_{\text {total }}$. Both $F_{0}$ and $F_{3}$ are multiplied by $-1, F_{1}$ and $F_{2}$ are multiplied by 1 , and $F_{4}$ and $F_{5}$ are multiplied by 0 . The expected value results in a polarization between -1 and 1 . In some situations, the most probable output may not match the ground state's output.

As just one example of why this more detailed analysis is necessary, we briefly discuss the simulations captured by Fig. 3 - a short wire segment with some device-level redundancy (the distance between redox sites is $1 \mathrm{~nm}$ and the center-to-center spacing between cells in the $\mathrm{x}$ or $\mathrm{y}$ dimension is $2 \mathrm{~nm}$ ). However, we also introduce a stray charge into this configuration. When this arrangement of cells was simulated with a 2-state statistical mechanical model, simulation results showed that the wire would behave as shown in Fig. 3a - i..e the binary 1 at the input would propagate to output with a near $100 \%$ probability. However, when the exact same simulation was run assuming a 6 -state model, the ground state of the system was that shown in Fig. $3 \mathrm{~b}$. Only when the stray charge was moved at least $0.66 \mathrm{~nm}$ away from the plane of the devices, did the ground state resemble Fig. 3a. While stray charges would most likely sit in the scaffold - i.e. below the plane of the active sites of the molecule - physically this may not cause much of a problem at the logic level. However, stray charges and other defects (rotated cells, shifted cells, etc.) might cause these degenerate states and they should be included for completeness. Clocked systems should also be considered.

\section{CIRCUITS AND 6-STATE MOLECULES}

Here, we present a statistical mechanical analysis of the fundamental circuit constructs needed for a functionally complete logic set and implementable interconnect. Specifically, 


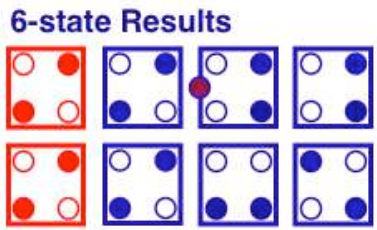

Figure 3: Defects may cause degenerate states.

we consider: (a) 6 active states, (b) ideal scaffolding dimensions, and (c) QCA molecules with reasonable dimensions ${ }^{2}$. DNA scaffolding dimensions will be considered in the next section. Majority gates, inverters, and wire segments, all a single cell "wide," are studied - enough to realize a functionally complete logic set and implementable interconnect. Redundancy at the device level is also addressed. Note that our simulations consider the full truth table for each construct. (The probabilities of a correct output associated with each input combination are averaged).

In Fig. 4, contour plots report the probability of a correct output for various circuit constructs as a function of centerto-center cell spacing. Inputs are at lower $\mathrm{x}$ values and outputs are at higher $\mathrm{x}$ values. Thus, computation flows along the x-axis from "left-to-right". All simulations assume room temperature operation (i.e. $300 \mathrm{~K}$ ).

Fig. 4 is organized as follows: Fig. 4a, c, and e report the probability of a correct output for a majority gate, inverter, and wire segment respectively. All constructs have no device-level redundancy and are thus one cell thick. We assume a distance of $0.9 \mathrm{~nm}$ between redox sites. Figures 4a-b, d-e illustrate how the probability of a correct output is affected as the center-to-center distance between devices changes, but for different sized molecules. Cell spacing in the $\mathrm{x}$ and $\mathrm{y}$ dimensions is plotted along the $\mathrm{x}$ and $\mathrm{y}$ axis; the probability of a correct output corresponds to the color of the plane. (Figs. $4 \mathrm{~b} / \mathrm{d}$ assume $1.5 \mathrm{~nm}$ redox spacing.) This study provides insight into how the distance between redox sites affects ideal scaffolding size. Fig. 4c/f provide insight as to how device level redundancy affects the probability of a correct output. Representative data for 1 and 3 cell wide wire segments is provided.

We discuss ideal scaffolding spacing first, and begin by noting that QCA molecules could be placed too closely together. For every circuit with cells in more than 1 row, there is a region in the contour plot (for low $\mathrm{x}$ and $\mathrm{y}$ values) that indicates a low probability of a successful output. An example is shown in the insert of Fig. $4 \mathrm{a}$-derived from assuming $0.9 \mathrm{~nm}$ redox spacing and $1.2 \mathrm{~nm}$ center-to-center cell spacing. Thus, there are just $0.3 \mathrm{~nm}$ between cell edges. If devices are placed too close together, the distance between redox centers is greater than the distance between device edges. The electrostatic coupling between redox centers of adjacent molecules is tighter than the coupling between the redox centers within the cells themselves, and charges have a tendency to "flee". Not surprisingly, as the distance be-

\footnotetext{
${ }^{2}$ Several candidate QCA devices have been made. The distance between redox centers for the molecule discussed in [9] is essentially $0.6 \mathrm{~nm}$. The distance between redox centers for the Creutz-Tabue ion is $0.9 \mathrm{~nm}$. Larger molecules have also been made. In an effort to "summarize" the range of spaces, we will consider 4-dot QCA molecules with $0.9 \mathrm{~nm}$ and 1.5 $\mathrm{nm}$ between redox sites in this section.
}

\begin{tabular}{|l|c|c|}
\hline Construct & Min. Space (nm) & Max. Space (nm) \\
\hline $1 \mathrm{x}$ Wire & $1.0 \times 10^{-9}$ & $2.5 \times 10^{-9}$ \\
1x Inv & $1.3 \times 10^{-9}$ & $2.1 \times 10^{-9}$ \\
1x Gate & $1.5 \times 10^{-9}$ & $2.3 \times 10^{-9}$ \\
\hline
\end{tabular}

Table 1: Ideal center-to-center cell spacings for logic family with $0.9 \mathrm{~nm}$ between redox sites.

tween molecules increases, the probability of a successful output also drops - as electrostatic interactions fall off as the distance between charges increases.

It should be fairly obvious that there is also a "sweet spot" (or ideal cell spacing) for each circuit construct. We consider this further by looking at the graphs in Fig. 4a, c, and e. The most important question to consider is whether or not the "sweet spots" for each fundamental circuit construct have any overlap. If so, this means that, at least theoretically, there is a "universal" scaffolding that should allow all of the constructs for a functionally complete logic set to function correctly. For the constructs in Fig. 4a, c, and e, the minimum and maximum center-to-center cell spacings (assuming a square grid) are reported in Table 1.

One can see that center-to-center spacings ranging between $1.5 \times 10^{-9}$ and $2.1 \times 10^{-9} \mathrm{~nm}$ should work well for every construct. However, placement does not have to be this precise. Uneven grid spacings can still provide correct outputs. To get more insight, we examined the raw data used to construct Figs. 4a, c, and e, and considered only the cell spacings that gave a probability of correct output that was above $85 \%$. More will be said about acceptable probabilities in the next section. In many cases, the grid spacings between successful gates, wires, and inverters does not have to be the same and can vary by at least $0.4-0.5 \mathrm{~nm}$ and still be above the $85 \%$ threshold $(1.5 \mathrm{~nm}$ in the $\mathrm{x}$ and $1.0 \mathrm{~nm}$ in the y dimension is an example).

There appears to be a rough linear relationship between the spacing between redox sites and ideal center-to-center cell spacings. The graphs in the first and second rows of Fig. 4 have similar shapes. For a molecule with $1.5 \mathrm{~nm}$ between redox sites, the ideal range for all circuit constructs is 2.1-2.9 $\mathrm{nm}$ assuming a square grid. Again, for many cases, spacings can vary by $0.4-0.5 \mathrm{~nm}$.

Increasing the device level redundancy also has a positive affect on the probability of seeing a correct output. This is more important when defects are introduced - and will be discussed in detail in future work. However, we do note here that the number of times that a wire segment (for example) produces the correct output (with high probability) increases as wire thickness increases.

The above results are important for two reasons. First, they show that for existing molecules, there is a scaffolding that will facilitate the correct operation of all of the components needed for a functionally complete logic set and interconnect. Second, while we can use this information to target an ideal scaffolding, we can also use these results to target an ideal molecule. (It may be easier to construct a device that conforms well to a more easily fabricated scaffolding.) We consider one candidate - DNA tiles - next.

\section{CIRCUITS, 6-STATE QCA MOLECULES, AND A DNA SCAFFOLDING}

Using DNA as a scaffolding for QCA systems is seemingly a natural fit - as it forms a "grid" that would allow for 


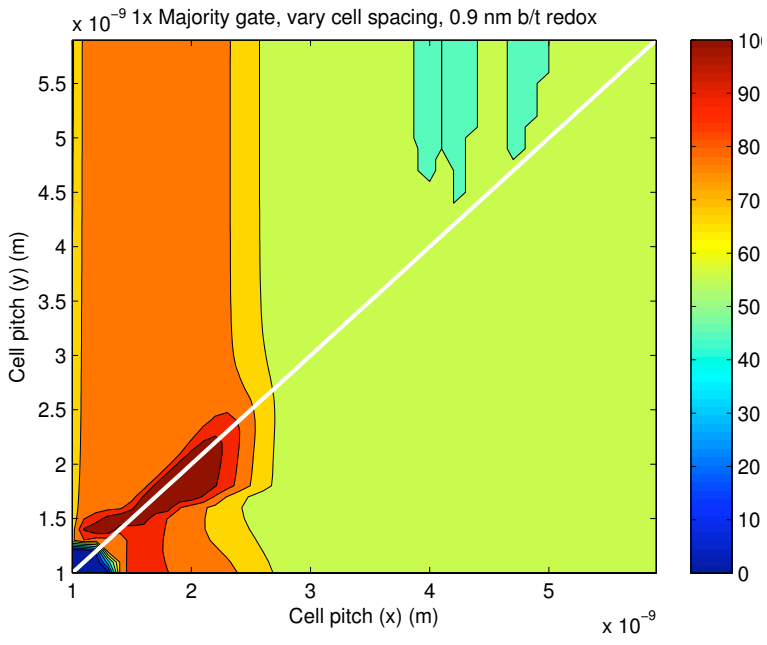

(a)

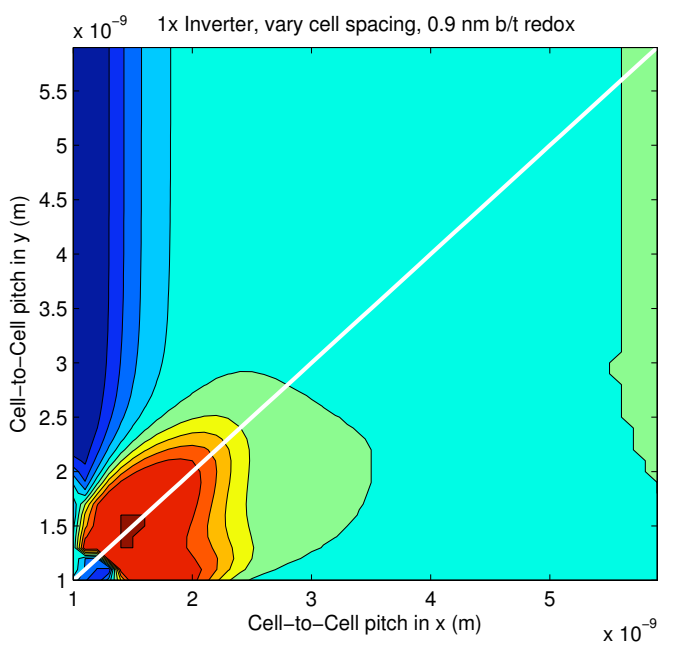

(c)

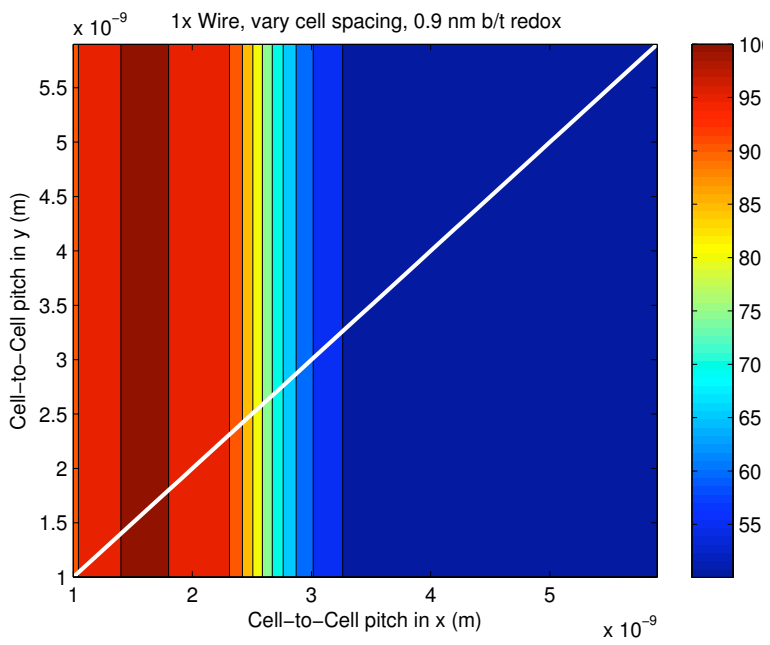

(e)

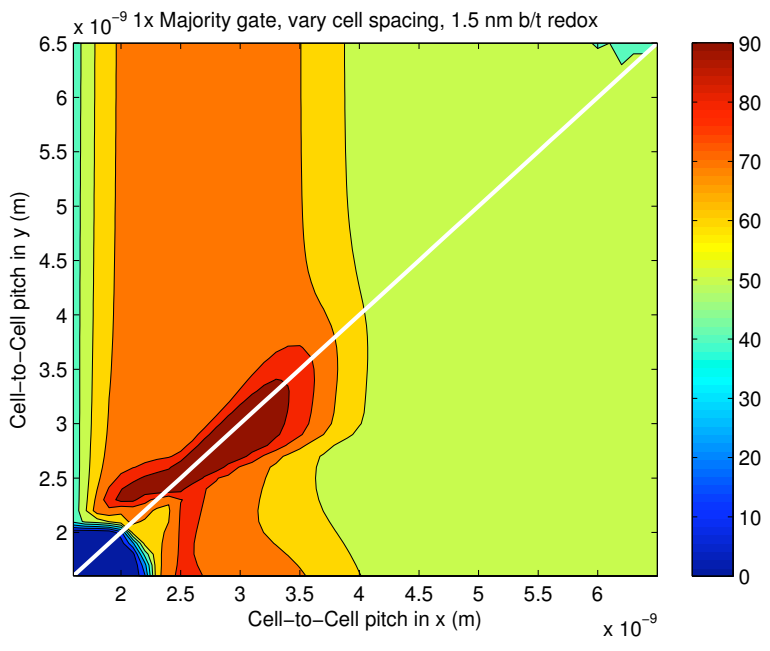

(b)

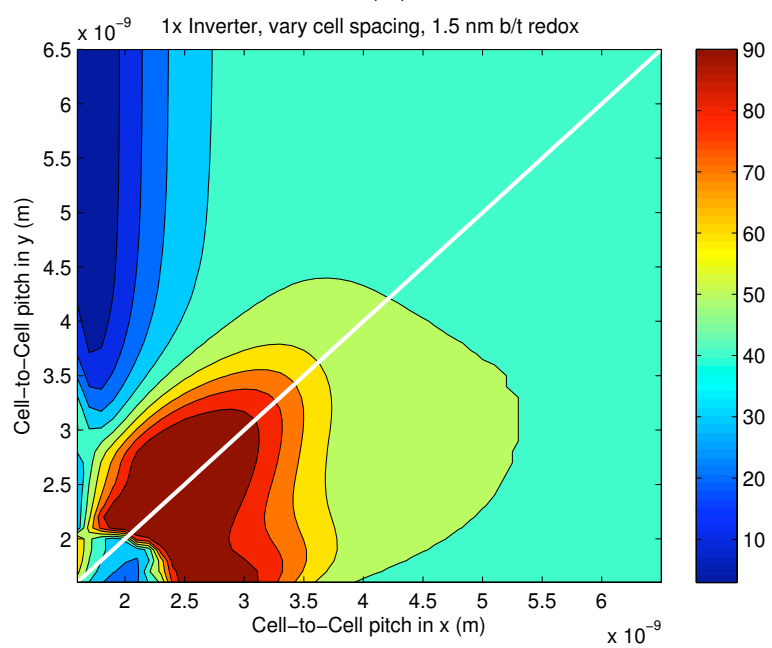

(d)

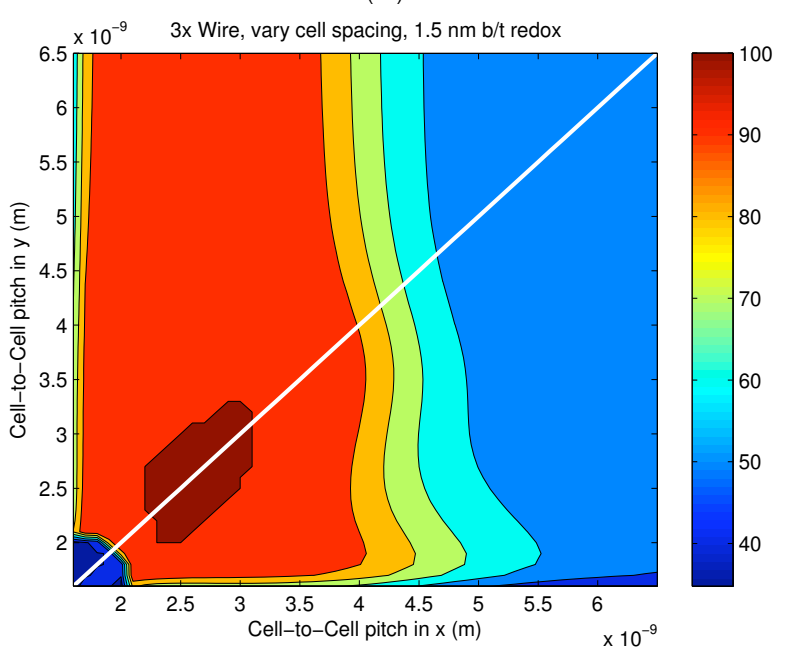

(f)

Figure 4: Probability of a correct logical output as a function of cell spacing. Graphs (a), (c), and (e) look at majority gates, inverters, and wires (all 1 cell thick) with $0.9 \mathrm{~nm}$ between redox centers. Graphs (b) and (d) look at majority gates and inverters for a larger molecule (1.5 nm between redox sites). Graph (f) looks at a wire segment 3 cells thick with $1.5 \mathrm{~nm}$ between redox sites. 
center-to-center spacings that are in the range of what appears to be ideal (a few $\mathrm{nm}$ ). However, DNA tiles may form a "grid" that is not square. Recall that groove sites may be $3.6 \mathrm{~nm}$ apart in the $\mathrm{x}$ dimension, and $2.0 \mathrm{~nm}$ apart in the $\mathrm{y}$-dimension. We will study how this affects a functionally complete logic set.

As our scaffolding attachment points are fixed, here we will vary the spacing between redox sites in an effort to determine the ideal sized molecule for this scaffolding. The probabilities with which wire segments and majority gates (with no device level redundancy and $2 \mathrm{x}$ redundancy) will produce the desired output are reported in Fig. 5a and $\mathrm{b}$ respectively. (Inverters were also simulated, but these results are not illustrated). Again, probabilities are summed and averaged over all input combinations.

Based on these simulation results we can make the following observations. First, it appears that smaller molecules (i.e. $0.9 \mathrm{~nm}$ between redox sites) and a DNA scaffolding with the aforementioned dimensions are not a good match. When the distance between redox sites is $0.9 \mathrm{~nm}$, the probability that a wire, inverter, and majority gate will produce the desired output ranges between 50-60\% - even with additional device-level redundancy. If we assume a molecule with $1.5 \mathrm{~nm}$ between redox sites, the probability of a wire segment functioning correctly increases significantly - to almost $100 \%$ for varying degrees of thickness, but only to 65 $70 \%$ for majority gates and inverters. (This makes sense as the dimensions of DNA are much closer to the ideal dimensions for the $1.5 \mathrm{~nm}$ molecules)

We believe that these lower overall probabilities occur for several reasons (depending on the construct and input combination). First, for certain majority gate input combinations (for example), the rigid DNA spacings results in a situation similar to that shown in Fig. 5a. Second, because grid spacings are not square, there can be a greater interaction between cells in one dimension, than cells in all dimensions.Third, for the smaller 4-dot molecule, as cell spacings increase, the electrostatic interactions decrease. Still, this does not mean that DNA should not be considered as a scaffolding for molecular QCA devices. From a systems stand point, there are still other things we must consider.

The first is to make the DNA grid more square. We may have little control over the spacing between groove sites, but we do not have to use every groove site either. If we use every other groove site in the y dimension, our grid will be much more square $-3.6 \mathrm{~nm}$ in the $\mathrm{x}$, and $4.0 \mathrm{~nm}$ in the $\mathrm{y}$ dimension. Also, note that here the variance between the $\mathrm{x}$ and $\mathrm{y}$ grid spacings is just $0.4 \mathrm{~nm}$ - results from the last section showed that circuits could be tolerant to this variation. The probability of a correct majority gate output, as a function of redox spacing, assuming a DNA scaffolding where every other grid spacing is used is also shown in Fig. 5b. As one can see, for a device with approximately $2 \mathrm{~nm}$ between redox sites, the probability of a correct output is nearly $100 \%$ for the majority gate.

We can also consider how these circuit constructs - using the smaller molecule and a DNA scaffolding - would function in the presence of a clock. In an effort to gain some insight into this system, we have simulated several short wire segments with (a) M-AQUINAS (assuming molecules with $0.9 \mathrm{~nm}$ between redox sites), (b) our 6-state statistical mechanical simulations, and (c) a 2-state statistical mechanical simulation. Results from the 2-state simulation are shown

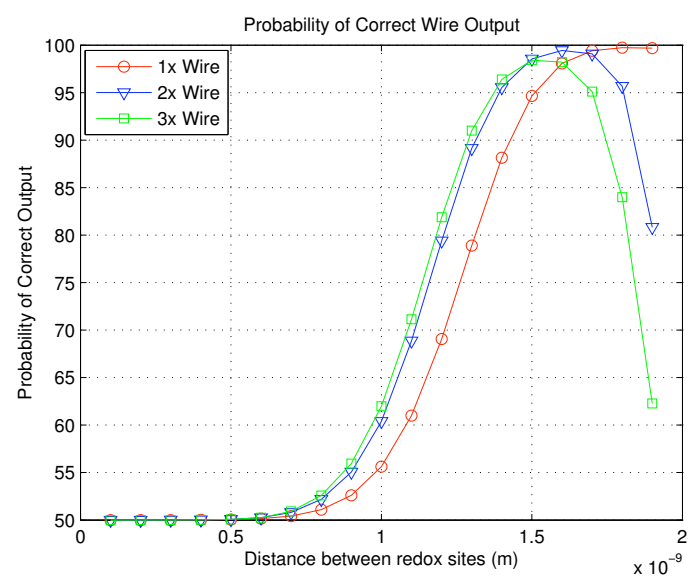

(a)

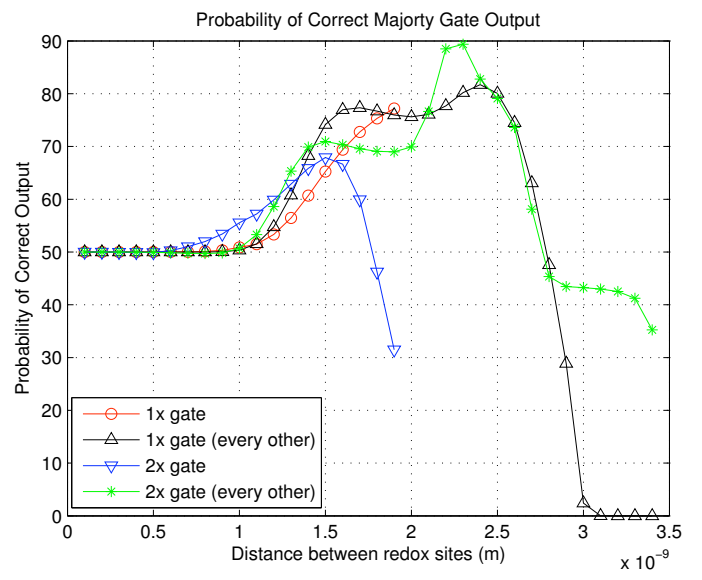

(b)

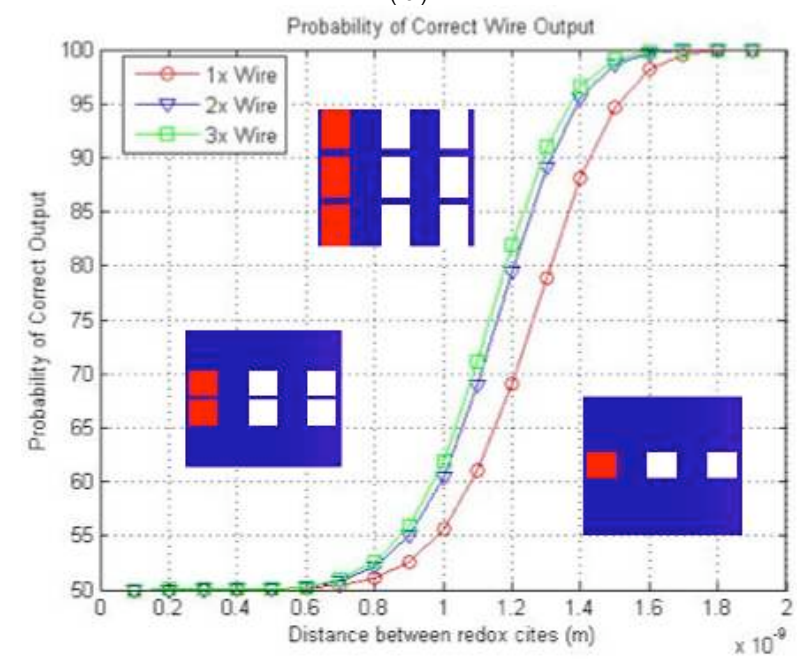

(c)

Figure 5: Probability of a correct output as a function of the distance between redox sites with a DNA scaffolding. (a) considers wires and $0.9 \mathrm{~nm}$ between redox sites, (b) considers majority gates using every DNA grid spacing, and every other grid spacing in the $y$-dimension, and (c) considers wires with a two state statistical mechanical simulation. Results from M-AQUINAS are inset. 
in Fig. 5c. Snapshots of the M-AQUINAS simulation setup are also shown as insets in Fig. 5c.

These simulation results show that for the single wide wire, a bit may not be transmitted successfully. However, for the $2 \mathrm{x}$ and $3 \mathrm{x}$ wires, a bit is transmitted successfully. Thus, it appears possible that - given the strong correlation between 2 and 6 state statistical mechanical simulations a clock could make a DNA scaffolding viable if the smaller molecule is used. Future work will further reconcile these results and will attempt to determine with what statistical mechanical probability a clock driven system will function.

\section{CONCLUSIONS}

This work not only creates a foundation for any other systems-level study of molecular QCA devices, but it also offers insights that are useful for physical scientists attempting to develop molecular QCA systems. It leverages a still evolving simulation suite to better account for mistake states in a molecular implementation of QCA. Results illustrate that we need to consider devices, scaffoldings, and circuits simultaneously - or we may run the risk of creating devices that work only in isolation. Experimentally, fabricating and placing just one device is a significant challenge. It makes sense to try to fabricate and place a device that will actually lead to circuits and systems.

The foundation created by this work will also inform computer scientists as to which implementation issues also require insight at the systems-level. For example, how will expected defects affect logic? Must architectures compensate? Will added device-level redundancy to combat defects add more switching events and degrade performance wins? Many of these questions only have answers at the systemslevel. Answering them will help determine the viability of an emerging technology. Future work will consider clocked, 6state systems, and further reconcile the differences between 2 - and 6-state simulations. Work that considers defects is already underway.

We thank NSF for supporting this work under CPA Grant 0541324 and Dan Gezelter for helpful conversations.

\section{REFERENCES}

[1] I. Amlani, A. Orlov, G. Snider, and C. Lent. Demonstration of a functional. quantum-dot cellular automata cell. J. Vac. Sci. Tech. B., 16:3795-99, 1998.

[2] P. Barbara, T. Meyer, and M. Ratner. Contemporary issues in electron transfer research'. J. Phys. Chem., 100:13148-13168, 1996.

[3] E. P. Blair. Tools for the design and simulation of clocked molecular quantum-dot cellular automata circuit. M.S. Thesis, Univ. of Notre Dame, 2003.

[4] A. Chaudhary, D. Chen, X. Hu, M. Niemier, R. Ravichandran, and K. Whitton. Eliminating wire crossings for molecular quantum-dot cellular automata implementation. ICCAD, pages 565-571, 2005.

[5] K. Hennessy and C. Lent. Clocking of molecular quantum-dot cellular automata. J. of Vac. Sci. \& Tech. B, 19(5):1752-55, Sep-Oct 2001.

[6] W. Hu, K. Sarveswaran, M. Lieberman, and G. H. Bernstein. High resolution electron beam lithography and dna nano-patterning for molecular qca. IEEE Trans. on Nano., 4:312-316, 2005.
[7] D. Hurley and Y. Tor. Ru(ii) and os(ii) nucleosides and oligonucleotides: Synthesis and properties'. $J$. Am. Chem. Soc., 124:3749-3762, 2002.

[8] A. Imre, G. Csaba, L. Ji, A. Orlov, G. Bernstein, and W. Porod. Majority logic gate for magnetic quantum-dot cellular automata. Science, 311, No. 5758:205-208, January 13, 2006.

[9] J. Jiao, G. Long, F. Grandjean, A. Beatty, and T. Fehlner. Building blocks for the molecular expression of quantum cellular automata. isolation and characterization of a covalently bonded square array of two ferrocenium and two ferrocene complexes. J. Am. Chem. Soc., 125:1522-1523, 2003.

[10] T. Labean, S. Park, S. Ahn, and J. Reif. Stepwise dna self-assembly of fixed-size nanostructures. Foundations of Nanoscience, Self-assembled Architectures, and Devices, pages 179-181, 2005.

[11] C. Lent and P. Tougaw. A device architecture for computing with quantum dots. Proc. of the IEEE, 85:541, 1997.

[12] M. Niemier and P. Kogge. Exploring \& exploiting wire-level pipelining in emerging technologies. In Proc. of Int. Sym. of Comp. Arch., pages 166-177, 2001.

[13] P. Perez-Tejeda, F. Sanchez-Burgos, and M. Galan. Calculation of rate constants from uv-vis spectroscopic data: an application of the marcus-hush model. J. of Molec. Struct. (Thermochem), 371:153-160, 1996.

[14] H. Qi, S. Sharma, Z. Li, G. Snider, A. Orlov, C. Lent, and T. Fehlner. Molecular quantum cellular automata cells. electric field driven switching of a silicon surface bound array of vertically oriented two-dot molecular quantum cellular automata. J. Am. Chem. Soc., 125:15250-15259, 2003.

[15] P. Rothemund. Design of dna origami. ICCAD, pages 471-8, 2005.

[16] P. W. K. Rothemund, N. Papadakis, and E. Winfree. Algorithmic self-assembly of dna sierpinski triangles. PLOS Biology, 2:2041-2053, 2004.

[17] G. Snider, A. Orlov, I. Amlani, G. Bernstein, C. Lent, J. Merz, and W. Porod. Quantum-dot cellular automata: Line and majority gate logic. Jpn. J. of Applied Physics, 38:7227-7229, 1999.

[18] J. Timler and C. Lent. Power gain and dissipation in quantum-dot cellular automata. J. of App. Phys., 91:823-831, 2002.

[19] G. Tom and H. Taube. The mixed valence state based on $\mu$-cyanogen-bis(pentaammineruthenium). J. of Am. Chem. Soc., 97:5310-5311, 1975.

[20] P. Tougaw and C. Lent. Logical devices implemented using quantum cellular automata. J. of App. Phys., 75:1818, 1994.

[21] K. Walus, V. Dimitrov, G. Jullien, and W. Miller. Qcadesigner: A cad tool for an emerging nano-technology. Micronet An. Work. 2003, Oct. 2003.

[22] Y. Wang and M. Lieberman. Thermodynamic behavior of molecular-scale quantum-dot automata (qca) wires and logic devices. IEEE Trans. on Nano., 3(3):368-376, Sept. 2004.

[23] E. Winfree, F. Liu, L. Wenzler, and N. Seeman. Design and self-assembly of two-dimensional dna crystals. Nature, 394:539-544, 1998. 